\title{
Laboreal
}

Volume $14 \mathrm{~N}^{\circ} 2$ | 2018

Digitalização e evolução do trabalho real

\section{La evolución digital y los cambios organizativos: qué respuestas de la ergonomía?}

A transformação digital e as mudanças organizacionais: que respostas dá a ergonomia?

Transformation numérique et changements organisationnels: quelles réponses de l'ergonomie?

The digital transformation and the organizational changes: which answers does ergonomics give?

\section{Sylvain Leduc y Ludovic Ponge}

\section{(2) OpenEdition}

\section{Journals}

Edición electrónica

URL: http://journals.openedition.org/laboreal/609

DOI: $10.4000 /$ laboreal. 609

ISSN: 1646-5237

\section{Editor}

Universidade do Porto

\section{Referencia electrónica}

Sylvain Leduc y Ludovic Ponge, «La evolución digital y los cambios organizativos: qué respuestas de la ergonomía? », Laboreal [En línea], Volume 14 №2 | 2018, Publicado el 01 diciembre 2018, consultado el 07 octubre 2019. URL : http://journals.openedition.org/laboreal/609 ; DOI : 10.4000/laboreal.609

Este documento fue generado automáticamente el 7 octubre 2019.

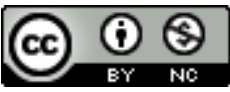

Laboreal está licenciado com uma Licença Creative Commons - Atribuição-NãoComercial 4.0 Internacional. 


\section{La evolución digital y los cambios organizativos: qué respuestas de la ergonomía?}

A transformação digital e as mudanças organizacionais: que respostas dá a ergonomia?

Transformation numérique et changements organisationnels: quelles réponses de l'ergonomie?

The digital transformation and the organizational changes: which answers does ergonomics give?

Sylvain Leduc y Ludovic Ponge

\section{NOTA DEL EDITOR}

http://dx.doi.org/10.15667/laborealxiv218sl

Manuscrito recebido em: julho/2018

Aceite após peritagem: outubro/2018

\section{Introducción}

1 En las empresas, la cuestión de la digitalización no es reciente, puesto que la introducción de las tecnologías de información y de comunicación (TIC) ocurrió hace muchos años. Sin embargo, con el desarrollo de la economía digital, se ponen de manifiesto nuevas cuestiones en relación al trabajo (Colin et al., 2015; Rosa, 2013). Según Roland Berger (2014), la Evolución Digital (ED) 'es una revolución industrial comparable a la que se vivió con la electrificación'. 
Desde los años 2000, las TIC se introducen gradualmente en el cotidiano de cada uno. Progresivamente, transformaron el ambiente socioeconómico y los comportamientos humanos, como lo había hecho la electricidad en su tiempo.

Esa transformación toca a la sociedad en su conjunto, y las empresas participan de ese movimiento de transformación. Por ejemplo, la ED transformó la relación con los clientes en el sector de los servicios, así como los diferentes modos de gestión. Actualmente, el interés por la ED es creciente, como lo muestra la progresión exponencial de las ocurrencias mediáticas sobre este tema en los últimos cinco años (Ilustración 1).

Ilustración 1: Media mensual de las ocurrencias mediáticas registradas por Google Trends $\AA^{\circledR}$ entre 2013 y 2018

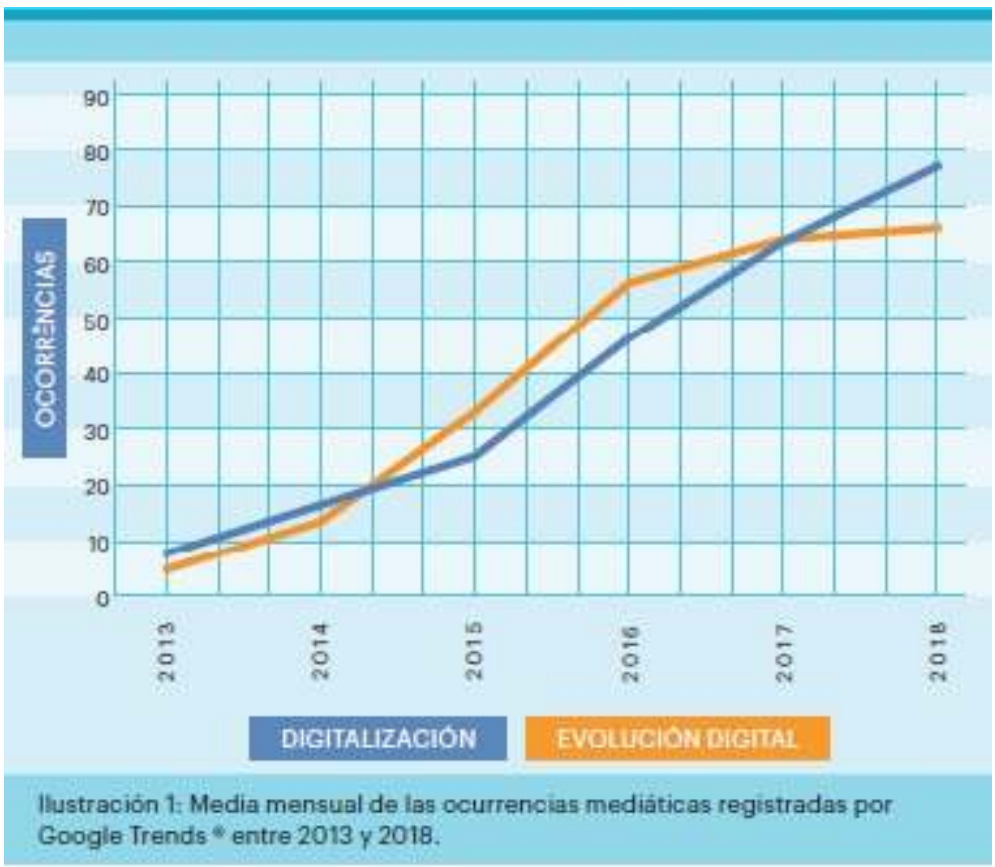

Hoy, La ED se impone a las empresas como un paso obligado. Por lo tanto, es esencial evaluar el impacto de esta evolución en el trabajo, relacionándolo con las mujeres y los hombres que lo ejecutan, ya que los cambios inducidos no parecen triviales.

Existe un volumen creciente de informes y documentos sobre los efectos de las TIC en el trabajo (DARES, 2018); su utilización está 'fuertemente correlacionada con una gran carga de trabajo y carga mental, así como con situaciones frecuentes de intromisión del trabajo en la esfera de la vida privada' (Elie-Dit-Cosaque, 2011). El desarrollo de las TIC a través de la ED se relaciona con el fenómeno de intensificación del trabajo que se inició desde la década de 2000. Las TIC se han convertido en socios 'indispensables' en el trabajo. Que sea a través de mensajerías, dispositivos móviles o paquetes de software de gestión, parecen generar nuevas obligaciones para los operadores. Contribuyen fuertemente al sentimiento de trabajar en un estado continuo de urgencia, que persiste en muchos de los trabajadores, incluso cuando la intensificación del trabajo parece haberse 'estancado' (DARES, ibid.). Sin embargo, la ED no es solo una cuestión de herramienta tecnológica, ya que se acompaña a menudo de nuevos métodos y formas de organización del trabajo. La 'fábrica del futuro' es uno de los muchos ejemplos, con el uso de tecnologías conectadas para mejorar la gestión de la producción y de los 
recursos. Köhler y Weisz (2018), especifican que el desarrollo de la industria que salga de la ED (industria 4.0) 'no significa más automatización o robotización... sino una nueva producción de flujos de información entre máquinas, personas, productos, usuarios'.

En el sector de los servicios, caracterizado por la producción de bienes intangibles, esta revolución se basa en la automatización del procesamiento de información, la integración de sistemas expertos para ayudar en las decisiones, o incluso la externalización de transacciones de bajo valor agregado, por medio de sitios web que ofrecen un espacio dedicado a que el cliente pueda administrar sus cuentas.

El propósito de este artículo es, por un lado, presentar los impactos de la ED en la actividad laboral a través de un enfoque sistémico y, por otro lado, identificar modalidades de transformación ergonómica. Como primer paso, se describen las diferentes formas de tratar la relación entre la ED y el trabajo, a partir de estudios relevantes sobre la ED en las empresas. Luego, a partir de casos prácticos, se estudian los efectos de la ED en la actividad laboral, utilizando los resultados obtenidos en dos intervenciones realizadas en diferentes contextos laborales. Para finalizar, se presenta una intervención para acompañar la ED que integra las exigencias de las situaciones de trabajo.

\section{Evolución Digital y el trabajo: cambiar de perspectiva}

8 La ED se encuentra al origen de profundas transformaciones del trabajo que no son neutras, ni ocurren por sí solas. Las empresas siguen esa tendencia por intermedio de importantes proyectos de inversión (industria 4.0, desarrollo de sistemas informáticos, rescate de start-up...) y de sus consecuentes reorganizaciones (nuevos procesos, nuevas herramientas, transformación de los oficios...). Se basan también en nuevas metodologías de desarrollo, de las cuales el UX Design es un ejemplo característico. Este método se basa en la experiencia del usuario ('User experience'), e intenta diseñar un producto que sirva a los usuarios finales, anticipando sus expectativas y necesidades. Esas transformaciones justifican interesarse al enfoque con el cual se entiende el trabajo en los procesos de cambio organizativo y tecnológico.

\subsection{De una mirada centrada en la tecnología que se impone a la actividad laboral...}

Los efectos de la ED han sido el objeto de numerosos informes (Lemoine 2014, Mettling 2015, Benhamou y Janin 2018), en los cuales las conclusiones oscilan entre las consecuencias en materia de intensificación del trabajo, o incluso de alienación, y una expectativa de mejora de las condiciones de vida en el trabajo. En este contexto, la digitalización es descrita como un factor de aceleración de transformaciones conduciendo a una mayor productividad. La única conclusión compartida se relaciona con las alternativas para la organización de las tareas y de los equipos. Además, los proyectos de ED habitualmente iniciados por las empresas se focalizan primariamente en la tecnología y en las funcionalidades y/o servicios que pueden aportar. En otras 
palabras, el progreso tecnológico se impone al operador con la finalidad de 'facilitarle la tarea' o incluso sustituirlo en su ejecución.

Esos enfoques' centrados en la tecnología' contribuyen globalmente a reducir el lugar del trabajador. Los enfoques denominados ‘UX Design' (User Experience Design o Diseño centrado en la experiencia del usuario) se desarrollan ampliamente, pero en gran medida se mantienen centrados en el usuario-cliente y no en el usuario-operador, lo que sigue dejando por fuera el trabajo real.

11 Las herramientas digitales se vuelven entonces actores de la actividad de trabajo y productores de los servicios. La función del operador se reduce a ejecutar fragmentos de tareas que no se pueden atribuir a la tecnología. El estudio de la gestión de estos proyectos 'tecnológicos' lo deja en evidencia sobre todo en la manera de considerar las exigencias de los operadores, describiéndolos más como 'utilizadores'. Por lo tanto, sus necesidades para realizar las tareas son a menudo descontextualizadas del trabajo real, incluso informados por usuarios de referencia (key-users) que no ejecutan las tareas en cuestión desde hace años, aunque son considerados como expertos. La usabilidad de las aplicaciones se limita al uso de normas que toman la forma de tablas ergonómicas estandarizadas, y que se deben emplear para definir los interfaces 'adecuados'.

\section{2. ... hacia una mirada centrada en lo humano a partir de la actividad laboral}

12 La comprensión de los desafíos laborales relacionados con la ED debe tomar en cuenta el conocimiento relativo a las actividades mediadas por herramientas. En este contexto, Rabardel (2005) propone el concepto de 'instrumento'. Esta noción se compone del artefacto (herramienta) que constituye 'los medios de su acción y no de sus objetos', así como de un esquema de utilización integrando una dimensión individual y una social (Rabardel, 1995). Por lo tanto, los instrumentos 'no son conceptualmente neutros, pero contienen una concepción del mundo que se impone de modo más o menos importante a sus usuarios...' Por lo tanto, es en el uso que la tecnología toma toda su magnitud.

Por consiguiente, para aprehender los desafíos de la ED, es necesario empezar por un abordaje en términos de utilización. Es ese cambio de perspectiva que proponen Beguin y Rabardel (2000), con la adopción de un punto de vista antropocéntrico y de desarrollo de la actividad instrumentada. Este enfoque luego exige integrar el tema del trabajo en los proyectos de transformación, más allá de la cuestión del factor humano. BobillierChaumon (2003) agrega que no hay determinantes positivos o negativos de las nuevas tecnologías, sino que los efectos dependen de la manera en que se piensan e implementan. Es decir, la representación que los directores del proyecto tienen de la posición y de la función del operador en el trabajo ejecutado, así como el conocimiento real de este trabajo.

14 Finalmente, el enfoque centrado en el operador es parte del desarrollo de lo que se designa como los entornos de trabajo 'habilitadores' que constan de tres características principales (Pavageau, Nascimento \& Falzon, 2007):

- Son preventivos, es decir que "no son perjudiciales para el individuo, ya que preservan sus capacidades futuras...';

- Son universales, es decir que son adaptados a las diferencias interindividuales y favorecen la integración, la inclusión y el reconocimiento social; 
- Tienen potencial de desarrollo, ya que permiten la adquisición de 'nuevas aptitudes y de nuevos conocimientos', favoreciendo la autonomía y el aprendizaje. conducidos en dos empresas de servicios. La finalidad de los proyectos era la revisión del servicio en respuesta a la evolución de las expectativas de los clientes. En ambos casos, la evolución tecnológica fue acompañada por un cambio organizacional en el servicio brindado con impacto en la organización del trabajo. En ambas empresas, las propuestas de servicio existentes se integran a nuevos sistemas técnicos y organizativos para gestionar las relaciones con los clientes de manera diferente.

En el caso del transporte aéreo, la demanda es hecha por la dirección para prevenir accidentes de trabajo, mientras que, en el caso del banco, son los representantes de los trabajadores quienes solicitan que se realice la intervención, como resultado de observaciones hechas por empleados.

\subsection{Las intervenciones y sus contextos}

Por razones de confidencialidad de las empresas de las cuales provienen los datos, solo se exponen elementos genéricos de las situaciones de intervención.

\subsubsection{Caso 1: El transporte aéreo}

Una aerolínea ha decidido desarrollar un nuevo dispositivo de información, combinando una tableta y un sistema de información, para ofrecer a los pasajeros un servicio en cualquier momento y en cualquier lugar del aeropuerto. El servicio propone un acceso en tiempo real al sistema de gestión de operaciones previas al vuelo. Esa nueva herramienta ofrece las siguientes funcionalidades:

- Información sobre las salidas y llegadas de vuelos para guiar los pasajeros y agilizar las escalas;

- Información sobre las características y el progreso del vuelo, así como las comidas servidas a bordo (con la posibilidad de especificar preferencias);

- La lista completa de los pasajeros de un vuelo, incluso los datos personales y el estatuto en el programa de fidelización;

- Información de conexiones de pasajeros, incluso detalles sobre irregularidades o retrasos;

- La venta de servicios adicionales, como el acceso al salón o compra de equipaje adicional;

- Un traductor y un directorio de todos los contactos útiles dentro de la empresa y en las escalas. 

anticipar sus necesidades y brindarles una atención especial, incluso 'personalizada'. También tiene como objetivo mejorar la vida cotidiana de los equipos al proporcionarles funcionalidades tales como mirar planes de vacaciones, tableros de instrumentos por vuelos, acceder a correos electrónicos, o participar en una red social profesional.

La intervención se originó a partir de la demanda del servicio de prevención de accidentes de la empresa. Se pidió un estudio de campo para evaluar los impactos del dispositivo en:

- La relación con el cliente;

- Las prácticas de trabajo;

- La prevención de riesgos laborales.

\subsubsection{Caso 2: El banco}

Como en el caso del transporte aéreo, el banco ha implementado tabletas con firma electrónica para acelerar las operaciones de apertura de cuentas o de suscripción a productos financieros. La eficiencia de este nuevo sistema requiere adaptar el sistema organizacional y la gestión del servicio brindado a las expectativas y comportamientos del cliente. Durante la última década, los clientes de los bancos han podido acceder a muchos servicios remotos, a distancia, al punto que ahora se encuentran de forma menos directa con los ejecutivos de cuenta y los solicitan cada vez más por correo electrónico o a través de los sitios web dedicados.

Este proyecto suponía la implementación de nuevos métodos de gestión en las relaciones con el cliente y, en última instancia, tenía como objetivo reducir la mano de obra en las agencias a través de un proceso de redefinición de los principios de evaluación de la carga de trabajo de los equipos. Todos los operadores se encontraban implicados en el proyecto, independientemente de sus ocupaciones (director, ejecutivos de cuenta, administrativos), experiencia, antigüedad o edad.

La intervención realizada es el resultado de una demanda del Comité de Salud, Seguridad y Condiciones de Trabajo (CHSCT) luego de un proyecto de reorganización, según lo autorizado por el código laboral francés a través de los artículos L 4612-8 y L 4614-12. Los representantes de los trabajadores cuestionaron el impacto de la implementación del proyecto, debido a la reducción de los puestos de trabajo que se produjo por los nuevos principios organizacionales y los nuevos métodos de gestión de clientes. Esta preocupación estaba ligada a comentarios de los trabajadores afectados ante las primeras etapas del proyecto. Expresan en particular, dificultades relacionadas con la nueva organización y la reducción de personal. Por lo tanto, la solicitud de una intervención del CHSCT se refiere a:

- Los impactos del proyecto sobre las condiciones de trabajo y los oficios;

- Los riesgos para la salud de los trabajadores asociados al proyecto;

- La gestión del cambio a través de módulos de capacitación y de seguimiento;

- La prevención de riesgos laborales.

Laboreal, Volume $14 \mathrm{~N}^{\circ} 2$ | 2018 


\subsection{Metodología} propias a la ergonomía y la psicología del trabajo, en particular entrevistas y observaciones en contexto y tiempo real. Se aplicaron métodos de implementación específicos según los contextos de intervención y características de las situaciones de trabajo. El objetivo fue identificar los determinantes del trabajo impactados por la ED, yendo más allá de una lógica centrada en la herramienta para adoptar un enfoque sistémico que considerase la interacción entre el factor humano y el sistema de trabajo en sus componentes técnicos y organizativos.

\subsubsection{Caso 1: El transporte aéreo}

La metodología de intervención realizada incluyó, por un lado, una recopilación de datos de campo a través de métodos cualitativos y cuantitativos y, por otro lado, la animación de talleres con agentes en las funciones impactadas por el proceso, para compartir y enriquecer los análisis de campo. La intervención tuvo una duración de 3 meses.

Los datos se recolectaron a través de entrevistas individuales con gerentes y operadores, así como observaciones $(n=50)$ de éstos en situación real de trabajo en diferentes aeropuertos $(n=6)$. También se distribuyó a todos los operadores un cuestionario sobre la aceptación de la tecnológica, con un gran volumen de encuestados $(n=900)$.

\subsubsection{Caso 2: El banco}

La metodología de análisis se fundó sobre tres principios conductores interdependientes:

- La caracterización de los cambios inducidos por el proyecto y el sistema de gestión de cambios previsto;

- El análisis de la actividad de recepción del cliente y la evaluación de los impactos relacionados con los cambios;

- La identificación de nuevas restricciones y recursos relacionados con el proyecto de ED para las actividades comerciales de las agencias.

Se recopilaron datos sobre los principios rectores del proyecto, los criterios y estándares utilizados para evaluar la carga de trabajo.

El campo de estudio se componía de en una docena de agencias en las cuales se realizaron encuestas (gerente de proyecto, gerentes de agencia, supervisores locales, agentes), así como observaciones del trabajo durante una sesión de un día $(n=50)$. La investigación se centró, en particular, en los efectos de la recepción al cliente compartida, el manejo de las ausencias, la articulación entre las dos funciones que debían cumplir los agentes (recepción y asesoramiento). También se distribuyó un cuestionario de salud ocupacional a los empleados que se encontraban en las agencias. Finalmente, se analizaron diversos documentos relacionados con la organización (balance social, descripciones de las ocupaciones, procedimientos...), así como los elementos utilizados para identificar las agencias pilotos (volumen de clientes, distribución de empleados por ocupación...).

Laboreal, Volume $14 \mathrm{~N}^{\circ} 2$ | 2018 
La intervención realizada tuvo lugar en varias fases durante unos dos meses. Después de una etapa de encuadre, la primera fase se centró en las encuestas, la segunda en observaciones de campo, y la última en el desarrollo de recomendaciones. Durante la intervención, se realizaron intercambios cruzados con los diferentes actores: gerentes de proyecto, CHSCT y gerencia. Informes intermedios se elaboraron para obtener reacciones de los actores a los elementos recopilados durante la intervención. El propósito de estos intercambios fue llevar los actores a un diagnóstico que fuese compartido.

\subsection{Los resultados de las intervenciones}

Después del análisis, los datos recopilados se interpretaron (Ilustración 2) con referencia al modelo de los 'cinco cuadros' (Leplat \& Cuny, 1974) que discierne, por un lado, los determinantes del trabajo, a través de las condiciones internas y externas de la actividad de trabajo. Por otro lado, discierne a través de la actividad de trabajo las consecuencias para el trabajador y el rendimiento general del sistema de trabajo. Este modelo de análisis apunta a la interdependencia entre los factores determinantes de la actividad de trabajo (condiciones de ejecución, características de los operadores), y sus efectos (sobre la empresa y los operadores).

34 La capacidad para llevar a cabo un trabajo de calidad, para desarrollar aptitudes y para participar en un colectivo de trabajo, se considera un vector del rendimiento y del desarrollo de la salud y el bienestar.

Este modelo ha sido ampliamente comentado. Aunque tiene sus límites (Falzon, 2013), sigue siendo, desde nuestro punto de vista, una cuadrícula de lectura relevante para analizar los procesos de cambio en el sector de los servicios. Aunque no tiene capacidad predictiva, o es muy limitada, es una herramienta de análisis y de difusión para comprender las situaciones de trabajo. En este caso, el modelo permite un análisis sistémico de los resultados de las dos intervenciones, mediante la identificación de los factores de causalidad que resultan de la ED, así como sus efectos y modalidades de regulación que provocan en la actividad de trabajo. 
Ilustración 2: Modelo de los cinco cuadrados (Leplat \& Cuny, 1974).

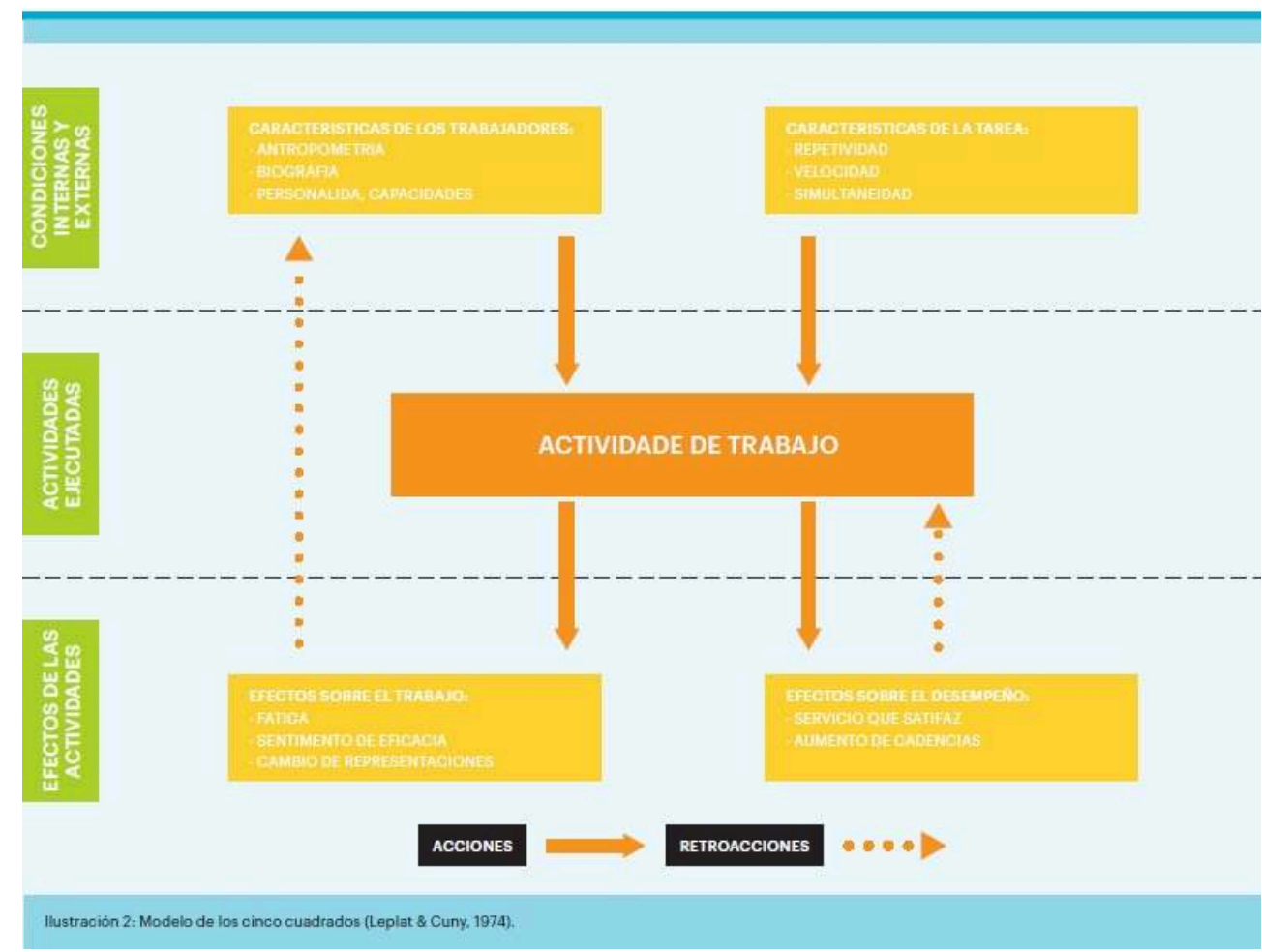

\subsubsection{LA ED y el cambio en los factores determinantes de la actividad de trabajo}

La ED ha inducido un cambio en el conjunto de los factores determinantes de la actividad de trabajo con consecuencias para la organización, sus relaciones internas y externas, así como para los propios operadores.

\subsubsection{Las consecuencias para la organización}

En el caso del transporte aéreo, los agentes ya no son asignados a una estación de trabajo fija y ven sus turnos de trabajo compuestos por una variedad de tareas, que se pueden desarrollar en diferentes lugares del aeropuerto según las necesidades de producción de servicios. La variabilidad de funciones y 'puestos' de trabajo es posible gracias a la naturaleza portable/móvil del dispositivo que permite el acceso al sistema de información en cualquier parte del aeropuerto. Es actualmente posible gestionar una relación con un pasajero independientemente de la ubicación (carriles de tránsito, sala de facturación, sala de embarque, rampa de acceso a la aeronave...) y de la solicitud (orientación, facturación de equipaje, cambio de vuelo...). En este caso, el efecto formador o 'capacitante' del dispositivo se basa en el 'nomadismo' que ofrece, aunque se han demostrado también las limitaciones tecnológicas relacionadas con la vida útil de la batería o la calidad de conexión móvil del dispositivo. Estas dos funciones son potencialmente limitantes para acceder al sistema de información, sin las cuales no se pueden realizar las tareas.

En el caso del banco, los cambios implementados en el marco del proyecto de ED modificaron las modalidades de repartición del trabajo. La gestión de la 'recepción del cliente', antes asegurada por un agente específico, se comparte hoy entre los ejecutivos o asesores según un proceso de rotación. Dado que las modalidades operativas no son 
definidas en el proyecto, se desarrollan diversas formas organizacionales según los equipos de trabajo (rotación de medio día o de todo el día...) y las opciones de gestión (participación de todos los agentes o solo de los ejecutivos o asesores a cargo de clientes privados). Sin embargo, algunos equipos encontraron modalidades operacionales más 'formadoras' que otras, como la implementación de cambios en la división de tareas por medio día, que han permitido conciliar las tareas de asesor con las de recepción. Estas modalidades han limitado los fenómenos de sobrecarga observados en los otros modos de operación, en particular al dejar la posibilidad de tratar sin demora las emergencias comerciales. También ayudan a mantener, o incluso desarrollar, competencias profesionales y amplía las relaciones con el trabajar en sus diversas dimensiones.

Todavía, la decisión de gestión del proyecto había sido de no tratar el tema, lo que impidió un debate sobre estos diferentes métodos de regulación.

Con respecto a los objetivos de resultados cuantitativos y cualitativos de los ejecutivos de cuenta - asesores, las observaciones revelan la existencia de impactos en relación a los indicadores utilizados, los cuales existen desde hace más de 10 años. Los objetivos de 'contactos' permanecen prácticamente sin cambios, aunque no se lograron en la modalidad organizativa anterior. Además, no se tomó en cuenta los trastornos generados por la ED sobre la calidad de la relación con el cliente, el 'servicio funcional' parece tener prioridad sobre la 'calidad relacional'.

\subsubsection{Las consecuencias para los servicios brindados}

En el caso del transporte aéreo, la supresión de la 'ventana' física acerca el agente del cliente, reduciendo las barreras entre el proveedor del servicio y su destinatario, y favoreciendo un servicio coproducido. Actualmente, el cliente puede tener información, que el agente no tiene, o que solo obtiene posteriormente por intermedio de otros dispositivos de información (por ejemplo, alerta de retraso de un vuelo transmitido en un teléfono inteligente). Así, para tratar su problema, el cliente tiene la expectativa de una pronta respuesta sobre esa información. Las competencias que deben tener los agentes se basan en la capacidad de explicar y aún más, de solucionar, aun cuando algunas 'claves de solución' no dependen de ellos, tampoco de su perímetro de acción. Por ejemplo, el caso de un retraso de vuelos que depende solamente de los servicios de control de tráfico aéreo.

En el caso del banco, con una transformación de las modalidades de contacto (aumento de solicitudes a distancia por teléfono y correo electrónico), la ED cambia los comportamientos. Asimismo, si los flujos de clientes en las agencias disminuyen, la ED genera otros tipos de contactos con demandas temporales similares. También se debe tener en cuenta que, en el marco de sus relaciones con los asesores, los clientes expresan una demanda cada vez más fuerte en cuanto a la reactividad temporal del asesoramiento, así como crecientes exigencias en términos de competencias esperadas en el asesoramiento. También ejercen progresivamente más un modo de concurrencia entre los actores bancarios. Por su parte, el banco reduce las horas de recepción para operaciones 'simples' e induce los clientes a utilizar los cajeros automáticos y las plataformas en línea. 


\subsubsection{La ED y sus consecuencias sobre los operadores}

43 Los cambios en los determinantes del trabajo afectan también a los operadores, con relación a sus competencias, la gestión de sus carreras profesionales, y el significado en el trabajo.

En el transporte aéreo, las exigencias de trabajo evolucionan desde el dominio de la gestión de pasajeros hacia la capacidad de emplear un dispositivo y sus funcionalidades en una situación de trabajo dinámica a nivel espacial, temporal y relacional. Los operadores encuentran dificultades de utilización que derivan de la disminución de su campo de acción con los clientes, y también se interrogan sobre su contribución en la empresa. En este escenario, se plantean nuevas preguntas a los supervisores sobre la asignación de recursos humanos 'limitados', cuando la fuerza de trabajo disponible ya registra una tendencia de disminución. Se asigna menos operadores a los equipos, y algunos de ellos no pueden ser enteramente operativos por falta de conocimiento sobre la utilización de esta nueva herramienta.

En el banco, los cambios afectan a las ocupaciones de manera diferente. Los puestos de asistente desaparecen, lo que requiere una conversión de los empleados afectados. Para los asesores y la gerencia, los cambios se caracterizan por la necesidad de dominar más tareas (especialmente las de recepción), la adquisición de nuevas competencias, una amplia versatilidad que reduce la capacidad de desarrollar experiencia, el cambio del significado del trabajo, y perspectivas menos estimulantes para el desarrollo profesional.

\subsubsection{Las consecuencias de la ED sobre la actividad de trabajo}

Ante estos cambios en los determinantes de la actividad, los operadores están sujetos a nuevas obligaciones que resultan de la aceleración del ritmo: aumento del número de clientes que deben gerenciar en forma consecutiva o incluso simultánea, lo que conduce a una interrupción y segmentación de tareas. El creciente número de productos/servicios y de las competencias pedidas hacen el trabajo más complejo. Estos cambios incrementan el envolvimiento del operador en su trabajo, con mayor movilización de recursos y exposición (al menos psicológicamente) al cliente, lo que tiene repercusiones sobre la salud mental en el trabajo y en el rendimiento productivo, como lo demuestran ciertos errores o estrategias de regulación implementadas.

En el caso del transporte aéreo, el personal ya no está asignado a un puesto y debe dominar diferentes posiciones de acuerdo con las necesidades de recursos humanos durante todo el turno. Esta versatilidad requerida se acompaña de una variación de situaciones, especialmente debido a las contingencias del transporte aéreo, y de los contextos de trabajo en respuesta a las necesidades de servicio esperadas e imprevistas. Los agentes deben estar capacitados para realizar una multitud de tareas independientemente del lugar y de la situación. Las situaciones se multiplican, y se necesita emplear herramientas con crecientes funcionalidades para tratarlas, lo que incrementa la complejidad. El trabajo real se vuelve más complicado.

En el banco, los operadores tienen que lidiar con múltiples demandas (clientes, tareas...) en un horizonte de tiempo cada vez más limitado. Este nuevo funcionamiento genera un incremento de las interrupciones del trabajo, que se agravan por una intensificación del trabajo 'digital', debido al creciente volumen de correos electrónicos 
de procesar, a la transferencia de llamadas telefónicas a las agencias, o a solicitudes de los clientes que son delegadas por el procesamiento de las aplicaciones digitales...

La nueva modalidad organizativa induce una doble actividad (recepción y gestión de carteras) que recae sobre la organización colectiva e individual del trabajo. La capacidad operativa de los asesores disminuye por la pérdida de control de su agenda y la reducción de tiempo para hacer frente a las demandas de los clientes. La gestión del rendimiento se supervisa cada vez más, basándose en objetivos de producción que pueden exceder las capacidades de los operadores y las realidades del mercado de demanda del cliente. Para algunos asesores, la multiplicidad de estos cambios conduce a una sobrecarga de información, con una disminución de la atención y una sensación de pérdida de control sobre su actividad de trabajo ('Puedo cometer errores sin darme cuenta'...).

Finalmente, la evolución digital modifica las prácticas comerciales en lo que toca a la gestión de relaciones con el cliente. Por ejemplo, con el surgimiento de negociaciones mediadas por teléfono o incluso por correo electrónico. En este último caso, esta modalidad asíncrona de comunicación revela sus límites, que se ilustran en la multiplicación de los intercambios para llegar a un compromiso, lo que resulta costoso en términos de tiempo como en términos de movilización cognitiva, esto haciéndose en desmedro de otras tareas que deben también realizarse.

\subsubsection{Las consecuencias de la ED en términos de rendimiento y de salud}

\subsubsection{Las consecuencias sobre la producción y la gestión del proveimiento de servicios}

51 En el caso del transporte aéreo, los clientes expresan ciertas expectativas que este nuevo dispositivo no puede satisfacer. El desarrollo progresivo de funcionalidades requiere el uso de estaciones informáticas estáticas, las cuales gradualmente son suprimidas. En algunos casos, la información suministrada por el nuevo sistema es errónea o en contradicción con el sistema de información anterior. Los agentes se enfrentan entonces a una disonancia cognitiva y social que requiere un arbitraje para completar o validar información, mientras tratan de mantener una conducta 'estoica' en la relación con el pasajero.

En el caso del banco, la reorganización de la recepción ha llevado a un aumento en el descontento y la rudeza de los clientes, aunque algunos se muestran más comprensivos y/o indulgentes. En este contexto, los empleados se expresan sobre la disminución en la calidad del servicio ('estamos estropeando el trabajo...'). Incluso reportan situaciones de deterioro de las reglas del trabajo (por ejemplo, rechazo de ventas: 'dije a un cliente que no podía hacer su dossier cuando lo podía, pero esto me hubiera tomado mucho tiempo').

53 Algunos asesores expresan la sensación de no poder hacer su trabajo correctamente ('no hacemos más nuestro trabajo con la misma capacidad'). Para los asesores, el proyecto de ED no apunta a la calidad del servicio, como se comunicó, sino a una lógica puramente financiera ('dirigirse à la recepción... no es para satisfacer el cliente...').

54 En los dos casos, el menosprecio por la actividad de trabajo tiene repercusiones en el rendimiento del sistema y la calidad del servicio prestado. Sin embargo, para el banco, el desempeño económico se logra a través de la reducción de personal y su consecuente reducción de costos, pero con un deterioro en la calidad del servicio. 


\subsubsection{Las consecuencias para los operadores, los equipos y la salud} de trabajo (necesidad de desplazarse, falta de soporte físico para usar el dispositivo ...). Para los agentes que no tienen un uso regular del dispositivo táctil, no solo pierden eficiencia en la realización de ciertas operaciones que normalmente se realizan en una estación de computadoras, sino que también son 'superados' gradualmente por las nuevas funciones implementadas cada mes. Esta herramienta de trabajo evoluciona regularmente debido a la modalidad de desarrollo utilizada, que se basa en un enfoque ágil, con entregas mensuales de nuevas herramientas. Estas dificultades se viven en forma diferente según los agentes. Es posible que un interés en estas nuevas tecnologías y una experiencia de su uso desarrollado fuera del trabajo pueda presentar una característica interindividual relevante para disminuir estas dificultades. Para los demás, puede ser reductivo considerar que la ausencia de estos elementos los lleva a enfrentar dificultades porque de acuerdo con las situaciones de trabajo (naturaleza de las solicitudes, misiones confiadas, trabajo colectivo), otras variables pueden acompañar la utilización de la herramienta. Por lo tanto, no es una cuestión de edad, de experiencia, sino de capacidades que pueden expresarse de acuerdo con los entornos 'capacitantes' en sus dimensiones sociotécnico-organizativas.

particular en agencias más pequeñas), el proyecto de ED genera una sensación de incertidumbre ante la percepción de no tener los medios para hacer lo que es ambicionado por los nuevos modelos organizacionales y/o por los clientes. A esto se añade una percepción de la reducción del tiempo comercial disponible y una sensación de mal uso de las competencias de cada uno: dos sentimientos que posiblemente podrían explicar un fenómeno de descualificación. Se les pide que 'pasen tiempo en la recepción en lugar de hacer su trabajo de asesoría'.

58 Las dificultades observadas afectan a todos los tipos de empleados, independientemente de la experiencia, el dominio del oficio o la edad. Además, hemos observado que los equipos de trabajo desempeñan un papel importante en el tratamiento de estas dificultades. Los equipos más solidarios generan una entreayuda que se traduce en regulaciones en la repartición de responsabilidades o de determinadas tareas, lo que se vuelve facilitador ante las nuevas molestias. Sin embargo, en algunas agencias las tensiones que resultan de la nueva organización son tan fuertes que la contribución del colectivo no es suficiente.

59 Las representaciones de los clientes sobre el nuevo funcionamiento ('¡Hoy están castigados!') a menudo se recibe negativamente y muchos asesores consideran que la contribución a la recepción es 'un paso atrás', puesto que, históricamente, la recepción es para los asesores la primera posición al ingresar a la empresa. En la industria aérea, la digitalización se describe como un factor de valorización profesional. Los pasajeros perciben que están delante agentes 'de vanguardia' con las últimas tecnologías, excepto cuando las mismas no están operativas. Por lo tanto, la valorización echa por los 
pasajeros a la tecnología aumenta la percepción de inutilidad hacia el agente: 'Tienes una herramienta y no puedes informarme!'; Una forma de incompetencia reconocida y reforzada.

En el sector bancario, la digitalización también debilita las relaciones de trabajo en los equipos. Surgen problemas en torno a la participación justa en el puesto de recepción. Las relaciones sociales con los representantes de la administración se deterioran debido a un enfoque de cambio que es percibido como viniendo 'de arriba abajo' y que deja poco espacio para escuchar la realidad experimentada 'en el frente operativo'. Esto es menos visible en el transporte aéreo porque los agentes pueden escalar/trasmitir sus necesidades de desarrollo técnico a través de la aplicación, sin todavía, tener la garantía de que esto se tendrá en cuenta. Una esperanza de evolución que puede seguir siendo vana, lo que contribuiría a un sentimiento de ser oídos sin ser necesariamente 'escuchados', entendidos.

61 En cuestiones de salud, el proyecto de ED genera en algunos empleados trastornos psicosociales que se exprimen en forma de crisis de llantos, perturbaciones del apetito y del sueño, incluso agotamiento. En el sector del transporte aéreo, las perturbaciones afectan principalmente a las relaciones laborales colectivas en forma de exclusiones sociotécnicas. Los agentes que no adoptan la herramienta se distinguen de los que la usan, como siendo relegados a la 'cola del pelotón'.

\section{Discusión: la ergonomía ante la ED}

Estos resultados muestran que la digitalización no es solo una evolución tecnológica de las herramientas de trabajo. Es ante todo una transformación radical del trabajo, tanto en su representación (relación con el trabajo) como en su realización (actividad real) Aunque los dos proyectos presentados sean diferentes, se identifican impactos similares en el trabajo de proveimiento de servicio. Sin embargo, la ED también puede constituir una base para desarrollar un entorno propicio para los operadores cuando las cuestiones del trabajo real están en el centro de la gestión del proyecto. Esto plantea varias preguntas relacionadas con la formalización del trabajo real y, por lo tanto, con el modelo de análisis a ser utilizado. Levanta también preguntas sobre la definición de los recursos de las situaciones capacitantes, en que se refiere a sus condiciones (factores externos de la actividad) y capacidades (factores internos).

Por ejemplo, en el caso del banco, el proyecto de ED define una prescripción en términos de gestión de la recepción, pero no estudia sus consecuencias a nivel operativo. Por lo tanto, aunque los flujos físicos de los clientes se reducen, los flujos generales no disminuyen para los asesores, sino que se transforman en aumento de solicitudes por teléfono y correo electrónico. Además, la carga de trabajo relacionada con la recepción sigue siendo importante, especialmente porque el proyecto de ED la ha condensado en espacios de tiempo más restringidos. Por lo tanto, la racionalización de los equipos que resulta de la nueva organización genera un fenómeno de intensificación del trabajo, con problemas para que los asesores respondan a los cambios en el comportamiento del cliente. Este fenómeno se ve acentuado por el hecho de que el proyecto no toma en cuenta que el número de empleados presentes en las agencias siempre difiere de la organización nominal (ausencias, vacaciones, etc.). Esto provoca que los periodos pasados en la recepción sean más largos que lo previsto en las evaluaciones de la carga de trabajo. 
En el transporte aéreo, la evolución de las molestias de naturaleza cognitiva, inducidas por el nuevo dispositivo, es poco conocida. Este tiene funciones similares a las de la estación informática fija, pero nada indica que los mecanismos intelectuales convocados para implementar una interfaz táctil en una situación de trabajo móvil sean idénticos. Seguramente, la atención necesaria no es la misma, una vez que los hitos circundantes difieren y que, el agente debe estar listo para contestar en cada momento las solicitudes de los pasajeros. Esto requiere de hecho una disponibilidad continua.

Por último, está la cuestión de los tiempos de apropiación y gestión de los cambios frente a una acumulación de evoluciones: cambios organizativos, nuevas herramientas de emplear (tableta, firma electrónica...). En el caso del banco, los empleados evidencian dificultades para apropiarse todos los cambios y herramientas, cuando el proyecto de ED postula que las nuevas herramientas con firma electrónica ya liberaron tiempo a los asesores.

Por lo tanto, el trabajo ocupa un lugar central para que los proyectos de ED constituyan entornos capacitivos para los operadores porque, por un lado, inducen cambios directos (caso del proveimiento de servicio) o indirectos (caso de los cambios organizativos) $\mathrm{y}$, por otro lado, modifican todos los determinantes del trabajo, impactando así a la actividad real.

67 No que se refiere al rendimiento, aunque se suponga un ahorro de tiempo, se concluye que los cambios observados (en la calidad de servicio, costes sociales o tiempo de producción) también lo requieren. Lo mismo ocurre con la salud: con un aumento en varias dimensiones de riesgo psicosociales, con cambios dentro de los equipos de trabajo, tensiones en las relaciones con los clientes, o un aumento en la intensidad de trabajo y del estrés. A esto, pueden agregarse, en ciertas situaciones, conflictos de valor que luego pueden alimentar diferencias en el trabajo, a menudo consideradas como contiendas entre personas.

Por lo tanto, para que la ED sea una oportunidad para mejorar las condiciones de trabajo, es esencial abordarla:

1. Integrando las cuestiones de trabajo hic et nunc, y no como debería ser en lo ideal;

2. Diseñando sistemas de trabajo 'capacitantes', y no solo sistemas de producción;

3. Repensando el diseño organizativo y el acompañamiento del cambio desde una perspectiva del trabajo real.

\subsection{Integrar los desafíos de la actividad de trabajo real}

69 La contribución histórica de la ergonomía es mostrar que el trabajo real difiere del que prescriben las organizaciones. Aunque es ampliamente reconocido, el análisis de la actividad de trabajo real se queda muy 'confidencial' en el contexto de proyectos de cambio. Estos proyectos son a menudo derivados de representaciones parciales de las realidades del trabajo, por ejemplo, considerando que la tecnología es infalible ('¡debe funcionar!'). Con frecuencia, algunas tareas se omiten por falta de conocimiento o de reconocimiento del trabajo que es solicitado 'en realidad' para garantizar el funcionamiento operativo.

70 Como lo demuestran los datos recolectados en las dos intervenciones presentadas, los cambios tecnológicos transforman fundamentalmente las condiciones de la actividad de trabajo, al modificar las reglas, los modos operativos de los agentes $\mathrm{y}$, en 
consecuencia, las regulaciones operadas por ellos. También pueden afectar la distribución de tareas, el funcionamiento de la estructura administrativa o los objetivos e indicadores de monitoreo de actividad. Por lo tanto, para el operador, estos cambios tienen múltiples efectos en sus competencias, su salud o en el significado que le da a 'su' trabajo.

71 Integrar el punto de vista de la actividad en el contexto de la ED consiste en pasar de un enfoque centrado en la tecnología, esencialmente encaminado por las funcionalidades de las herramientas, a un enfoque centrado en el operador (Béguin \& Rabardel, 2000), hincado en las utilizaciones de las herramientas y sus fundamentos cognitivos.

Considerar el tema de la actividad de trabajo real es esencial para comprender cómo los operadores podrán hacer frente a nuevas restricciones, generar nuevas regulaciones en su actividad, adaptarse a los cambios en el proveimiento de servicio, y apropiarse nuevas herramientas y tareas.

73 Analizar la actividad de trabajo implica percibir el 'factor humano' en sus aspectos fisiológicos, cognitivos, psicológicos y sociales, teniendo en cuenta el trabajo y sus varias 'producciones'. Esta comprensión es esencial para garantizar una adaptación óptima del trabajo al 'operador', en particular en un enfoque basado en la fiabilidad organizativa, según lo cual a menudo esto se considera el 'eslabón débil'. Desde la revolución industrial, numerosos accidentes han marcado la historia con ejemplos de sistemas inadecuados a las características de los 'operadores' que deben conducirlos. Caroly (2007) recuerda que "la inobservancia de la variabilidad individual frente a los cambios tecnológicos y a la progresión en el aprendizaje es el origen de sufrimiento y el deterioro de las relaciones interpersonales'(p. 5).

El análisis de la actividad de trabajo en su entorno encierra también ocuparse de los procesos de regulación en el trabajo. En este marco, la ergonomía desarrolló un modelo de regulación para describir las modificaciones de los modos operativos según sus restricciones (Guérin et al., 2001). Sin embargo, uno del interés de la comprensión de la actividad de trabajo hoy es poder construirse un modelo de actividad humana que propone, por un lado, articular las diferentes dimensiones que la componen y, por otro lado, distinguir 'su peso' relativo en esta actividad. De hecho, los cambios tecnológicos revelan la influencia de múltiples factores en el trabajo real, lo que hace difícil determinar lo qué 'pesará' más en las regulaciones de la actividad así generadas.

\subsection{Diseñar sistemas de trabajo 'capacitantes'}

75 Como Béguin (2007) nos recuerda, con demasiada frecuencia 'durante la concepción, existe una desproporción entre el cuidado que se toma en la fabricación de máquinas o la definición de organigramas y la atención prestada a quienes garantizan su operación diaria, a través de su trabajo'(p. 107). Es esta incongruencia que se refleja en los efectos de la ED.

Esta es la misma situación que revelan los casos presentados. Los proyectos están bien definidos en los principios, los indicadores de control, pero los métodos de configuración permanecen basados en la prescripción, por lo tanto, alejados de la actividad de trabajo y con una débil capacidad de ajustamiento frente a los resultados de experiencias que provienen de las realidades de campo. 


$$
\begin{aligned}
& \text { que se basa el diseño organizativo del trabajo. Al igual que los elementos presentados } \\
& \text { en el Informe Mettling (2015), los ejemplos anteriores muestran que: }
\end{aligned}
$$
en el Informe Mettling (2015), los ejemplos anteriores muestran que:

- la carga de trabajo no solo está relacionada con el tiempo, pero está también estrechamente relacionada con las exigencias de la actividad de trabajo (interrupciones, solicitudes múltiples, tareas dobles...);

- cada vez se realizan más tareas fuera de la presencia física del cliente, a pesar de que sea un coproductor vital del servicio;

- las tareas más simples son realizadas directamente por los clientes, quedándose las más complejas y lentas para los operadores, lo que reduce la variedad del trabajo y, especialmente, las fases de recuperación cognitiva;

- nuevas tareas, en particular las que se relacionan con las nuevas herramientas, se agregan, aunque el tiempo de trabajo permanece lo mismo;

- los indicadores de gestión tradicionales ya no son apropiados y, a menudo, no pueden informar sobre la actividad de trabajo real.

\subsection{Repensar los diseños organizacionales y el seguimiento del cambio}

80 Integrar en una de las primeras fase de los proyectos la cuestión de la 'probable actividad futura' (Daniellou, 2004, 2007) es pasar de un enfoque donde el trabajo es solo un medio de producir bienes o servicios (una variable de ajuste), a un enfoque en el cual el trabajo está en el centro del sistema productivo, además de la máquina. Esto supone ir más allá de una representación del trabajo como un costo que debe reducirse, para considerarlo como un recurso de producciones múltiples. Es el medio de 'actuar sobre' la inestabilidad intrínseca del sistema productivo (Dejours, 2003), conciliando la complejidad y la multiplicidad de exigencias.

81 Estos elementos son aún más significativos en el contexto del proveimiento de servicio ya que, como nos recuerdan Hubault y Bourgeois (2002), la característica principal de las situaciones de servicio es la irrupción de la 'subjetividad en el campo económico', refiriéndose, por ejemplo, a la irracionalidad en la confrontación entre la lógica de las necesidades con la lógica del derecho comercial. En los casos presentados, la negligencia de la actividad futura tiene impactos en el servicio, en particular en su calidad, incluso en situaciones de deterioro de las reglas del oficio (rechazo de venta en el caso del banco).

82 En cuanto al acompañamiento del cambio, desde el punto de vista del trabajo, Bobillier Chaumont y Clot (2016) subrayan que los empleados no permanecen pasivos ante las

Laboreal, Volume $14 \mathrm{~N}^{\circ} 2$ | 2018 
evoluciones. Por lo tanto, es necesario dialogar 'alrededor y sobre' la actividad de trabajo. Sin embargo, históricamente el acompañamiento del cambio se trata desde el punto de vista de la 'resistencia al cambio', lo cual debe reducirse o eliminarse a toda costa a través de un ejercicio de convicción hacia los agentes, argumentando como sea una necesidad 'dogmática' portadora de 'mejora'. La curva de duelo utilizada por France Telecom durante su transformación al final de la década de 2000 es un síntoma de este tipo de enfoque (Michaux 2018). En esta perspectiva centrada sobre la tecnología, el trabajo sigue siendo exógeno a la cuestión del cambio y el operador debe adaptarse a lo que es prescrito, lo que también ilustra un enfoque de trabajo como lo que 'debe ser'.

Incluso si 'los cambios rara vez tienen que ver con el trabajo' (Hubault, 1997, p. 91), él está en el centro de los desarrollos en las empresas y especialmente en lo que toca la ED. Por lo tanto, los cambios inducidos por la ED requieren también dialogar sobre el tema de la 'calidad del trabajo'. Al respecto, Clot (2013) afirma que ' lo que uno no puede hacer es tan importante como el de lo que se hace'(p. 25). Esta necesidad es aún más fuerte en situaciones de servicio, ya que Petit y Dugué (2013) señalan que 'la relación subjetiva desarrollada por los operadores con el trabajo se basará principalmente en su capacidad para producir un trabajo que los clientes consideren de alta calidad '(p. 1). En otras palabras, no solo se trata de coproducir un servicio, sino también de la calidad que lo 'califica' y permite apreciarlo en ambos lados del 'contador'.

Integrar la subjetividad en el acompañamiento del cambio, con cuestiones sobre el significado y la calidad del trabajo, es fundamental para la aceptabilidad de las ED. En última instancia, para que un nuevo sistema sea aceptable, debe 'tener suficiente adecuación con el usuario' (Dubois \& Bobillier-Chaumon, 2009). En el caso de los servicios, es necesario agregar '... y el cliente'.

\section{Conclusiones}

El interés de la ED en el campo de las condiciones de trabajo está directamente relacionado con cuestiones de los riesgos psicosociales y el deterioro de las relaciones en el trabajo. La ED no es solo una transformación de herramientas. Sus repercusiones apuntan a otro enfoque, en el cual 'los proyectos de transformación son una oportunidad ideal para actuar sobre todos los factores determinantes del trabajo y fomentar el desarrollo de la prevención' (Ponge \& Dugué, 2017 p. 18). Por lo tanto, es esencial cuestionar tanto las formas de integrar la prevención en los proyectos de ED, como el lugar que se puede dar a los ergónomos en esto tipo de cambio.

\subsection{La ED es mucho más que un cambio de herramientas}

Los efectos de la ED van mucho más allá que la cuestión de las herramientas tecnológicas. Es una 'digitalización de la organización del trabajo' que sobrepasa lo que las empresas han experimentado con la introducción de las primeras automatizaciones, como el control numérico. Hoy, hay sobreposición e inclusión de sistemas organizativos de la producción y del trabajo, cuyo ajuste depende del factor humano. A menudo se considera este factor a través de su capacidad de adaptación. El trabajo se encuentra totalmente 'transformado', lo que implica que, para diseñarse condiciones de trabajo 
sostenibles para las personas, se requiere replantear el enfoque de la ED en empresas y organizaciones.

\subsection{La prevención en el contexto de la ED}

87 En el contexto de la ED, la prevención primaria es necesaria para no quedarse en un enfoque únicamente curativo en las transformaciones de trabajo. Esto requiere la capacidad de influir en la gestión de los proyectos. La prevención de riesgos laborales se vuelve más efectiva si se integra lo antes posible en proyectos de cambio (Grosjean \& Neboit, 2000).

En su implementación, la ED usualmente toma la forma de gestión de proyectos. Sin embargo, como indica Midler (1996), los proyectos se caracterizan por un fenómeno de irreversibilidad: en el anteproyecto 'podemos hacer todo, pero no sabemos nada', mientras que al final 'lo sabemos todo, pero hemos agotado todas sus capacidades para la acción'. Por lo tanto, es necesario que las preguntas de prevención estén presentes en la gestión del proyecto para que se conviertan en una lógica intrínseca del proyecto (confrontadas, por supuesto, con otras lógicas también implicadas en el proyecto con las cuales se debe debatir y a las cuales se deberá adaptarse). Este aspecto también recuerda el valor agregado de la ergonomía del diseño como forma de desarrollar la prevención primaria.

\subsection{El lugar dado a la ergonomía y a los ergónomos en proyectos de ED}

Dados las desafíos relativos al trabajo, la ergonomía como una 'disciplina científica que busca una comprensión fundamental de las interacciones entre los humanos y otros componentes de un sistema...' (IEA - 2000) puede tener un lugar en los procesos de ED. Pero la ergonomía sigue siendo poco solicitada por los diseñadores y dirigentes, debido a sesgos cognitivos como la subestimación de los riesgos (profesionales) y la sobreestimación de las capacidades de adaptación del factor humano.

La comunidad de ergonomía tiene ventaja en la renovación de los debates sobre la representación de la profesión y sus contribuciones, en relación con los diseñadores y dirigentes. Dul et al. (2012) también han expresado la necesidad de los ergonomistas desarrollen sus comunicaciones con gerentes y tomadores de decisiones. Pero esto también implica cuestionar las competencias de los ergónomos en estos contextos, así como las representaciones de la profesión que transmiten o que se les atribuyen.

\section{BIBLIOGRAFÍA}

Béguin, P. (2007). Prendre en compte l'activité de travail pour concevoir. @activités, 4(2), 107-114. 
Béguin, P., \& Cerf, M. (2004). Formes et enjeux de l'activité pour la conception des systèmes de travail.@activités, 1(1), 54-71.

Béguin, P., \& Rabardel, P. (2000). Concevoir pour les activités instrumentées. Revue d'intelligence artificielle, 14, 35-54.

Benhamou, S., \& Janin, L. (2018). Intelligence artificielle et travail, Rapport à la ministre du Travail et au secrétaire d'État chargé du Numérique.

Bobillier-Chaumon, M-E. (2003). Évolutions techniques et mutations du travail : émergence de nouveaux modèles d'activité, Le Travail Humain, 66, 161-192.

Bobillier-Chaumon, M-E., \& Clot, Y. (2016). Clinique de l'usage: Les artefacts technologiques comme développement de l'activité, @activités, 13(2).

Caroly, S. (2007). Les mutations du travail face aux défis technologiques : quelles incidences sur la santé ?, Perspectives interdisciplinaires sur le travail et la santé, 9(2).

Casilli, A. (2014). Digital Labor: travail, technologies et conflictualités, pp, 10-42

Clot, Y. (2013). L'aspiration au travail bien fait. Le journal de l'École de Paris du management, 99.

Colin, N. et al. (2015), Économie numérique. Les notes du Conseil d'analyse économique, $n^{\circ} 26$.

DARES (2018). Quels liens entre les usages professionnels des outils numériques et les conditions de travail ? Analyses n029.

Daniellou, F. (2004). L'ergonomie dans la conduite de projets de conception de systèmes de travail. In P. Falzon (Ed.). Ergonomie. pp. 357-373. Paris: PUF.

Daniellou, F. (2007). Des fonctions de la simulation des situations de travail en ergonomie. @activités, 4(2), 77-83.

Dejours, C. (2003). L'évaluation du travail à l'épreuve du réel. INRA Éditions.

Dubois, M. \& Bobillier-Chaumon, M-E. (2007). Développement de l'expertise des usagers via les TIC: quels enjeux pour les travailleurs des relations de services ?, Pistes: Perspectives interdisciplinaires sur le travail et la santé, 9(2).

Dul, J., Bruder, R., Buckle, P., Carayon, P., Falzon, P., Marras, W., Wilson, J. \& van der Doelen, B. (2012). A strategy for human factors/ergonomics: developing the discipline and profession, Ergonomics, .1-27.

Elie-Dit-Cosaque, C. (2011). Les technologies de l'information et de la communication (TIC) et le contenu du travail, Annales des Mines - réalités industrielles, pp. 35-39.

Falzon, P. (2013). (Dir.) Ergonomie constructive. Paris: PUF

Grosjean, J.C., \& Neboit, M. (2000). Ergonomie et prévention en conception des situations de travail. In Cahiers de notes documentaires, Hygiène et sécurité du travail, 179. ND 2127. INRS.

Hubault, F. (1997). La place du travail dans les processus de changement. In J.-C. Crescenzo (Ed.) (2005) Changement dans les organisations, tome 1 stratégies, processus et performances. Paris : L'Harmattan, pp. 91-101.

Hubault F., \& Bourgeois F. (2002). La relation de service: une convocation nouvelle pour l'ergonomie? In F. Hubault, La relation de service, opportunités et questions nouvelles pour l'ergonomie. Toulouse. Éditions Octarès, pp. 5-31.

Kohler, D. \& Weisz, J.D. (2018), Industrie 4.0, une révolution industrielle et sociétale. Futuribles, 47-68. 
Lemoine, P. (2014). La nouvelle grammaire du succès. La transformation numérique de l'économie française. Rapport au gouvernement français.

Leplat, J. \& Cuny, X. (1974). Les accidents du travail. Paris: PUF.

Mettling, B. (2015). Transformation numérique et vie au travail, Rapport à l'attention du ministre du Travail, de l'Emploi, de la Formation Professionnelle et du Dialogue Social.

Midler, C. (1996). Modèles gestionnaires et régulations économiques de la conception. In G. (de) Terssac, \& E. Friedberg (Ed.). Coopération et Conception. Toulouse: Octarès, pp. 63-85.

Pavageau, P., Nascimento A., \& Falzon, P. (2007). Les risques d'exclusion dans un contexte de transformation organisationnelle. Perspectives interdisciplinaires sur le travail et la santé, 9(2).

Petit, J., \& Dugué, B. (2013). Quand l'organisation empêche un travail de qualité : étude de cas; Perspectives interdisciplinaires sur le travail et la santé, 15(2).

Ponge, L., \& Dugué, B. (2017), L'enjeu de la prévention primaire lors des projets de changement: quelle place pour le CHSCT? Quels apports de «l'expertise»?, @ctivités, 14(1).

Rabardel, P. (1995). Les hommes et les technologies, une approche cognitive des instruments contemporains, Paris: Armand Colin.

Rabardel, P. (2005). Instrument, activité et développement du pouvoir d'agir. In Philippe Lorino \& Régine Teulier. Entre connaissance et organisation: l'activité collective (pp. 251-265). La Découverte.

Berger, R. (2014). Du rattrapage à la Transformation, L'aventure numérique, une chance pour la France, sept. 2014.

Rosa, H. (2013). Mouvement historique et histoire suspendue. Vingtième Siècle, Revue d'histoire, 117, $89-104$.

\section{RESÚMENES}

La evolución digital (ED) produce importantes cambios organizacionales en las empresas, que impactan en el trabajo y sus condiciones de ejecución. Sin embargo, los cambios observados se abordan, o bien desde un enfoque centrado en la tecnología, donde 'la herramienta' aparece como la manera de resolver todos los problemas del mundo del trabajo, o bien siguiendo una orientación de paliativos que promueve la calidad de vida en el trabajo.

A partir de datos de campo de intervenciones ergonómicas realizadas en dos empresas de servicios, el artículo se propone mostrar cuales son los factores determinantes del trabajo que evolucionan con el efecto de la conjunción de varios cambios relacionados con la ED.

Los resultados destacan la necesidad de modificar los enfoques tradicionales de los proyectos de cambio tecnológico. El artículo luego propone respuestas sobre la conducción de estos proyectos, el diseño de los aparatos técnico-organizativos y las modalidades de acompañamiento del cambio.

Nas empresas, a Transformação Digital (TD) é acompanhada por grandes mudanças organizacionais com consequências para o trabalho e suas condições de realização. No entanto, estas mudanças são tratadas no âmbito de abordagens centradas na tecnologia, segundo as quais a 'ferramenta' é tida como a solução para todos os problemas do mundo do trabalho, ou por meio de uma abordagem paliativa que proclama a qualidade de vida no trabalho. A partir de dados de terreno derivados de intervenções ergonómicas realizadas em duas empresas de serviços, este artigo propõe-se a demostrar que é o conjunto de determinantes do trabalho que evolui sob o efeito da interação entre várias mudanças relacionadas com a TD. Os resultados destacam a 
necessidade de modificar as abordagens convencionais, segundo as quais se regem projetos de mudança tecnológica. $O$ artigo propõe alternativas para a condução desses projetos, para a conceção dos artefactos técnico-organizacionais, e para as diferentes formas de acompanhar a mudança.

Dans les entreprises, la Transformation Numérique (TN) s'accompagne d'évolutions organisationnelles majeures qui ont des impacts sur le travail et ses conditions de réalisation. Toutefois, les changements constatés sont soit abordés à partir d'une approche techno-centrée où 'l'outil' apparaît comme LE moyen de résoudre tous les problèmes du monde du travail, soit via une approche palliative prônant la qualité de vie au travail.

À partir de données de terrains issues d'interventions ergonomiques menées dans deux entreprises de service, l'article propose de montrer que c'est l'ensemble des déterminants du travail qui évolue sous l'effet de la conjonction des divers changements liés à la TN.

Les résultats soulignent la nécessité de modifier les approches traditionnelles des projets d'évolution technologique. L'article propose alors des réponses sur la conduite de ces projets, la conception des artefacts technico-organisationnels et les modalités d'accompagnement du changement.

In the companies, the Digital Transformation (DT) implies several organizational changes with consequences on effective tasks and working conditions. However, these changes are analyzed under the scope of technology-centered approaches, where the 'tool' is considered the main solution to solve all work-related problems or by a human-direct approach focused on quality of life at work. This paper aims to show that the work determinants evolve under the effect of the interactions among several changes related to DT. The findings are based on two field studies about practical ergonomics interventions in two different services companies. The results underline the need to change conventional approaches ruling projects of technological change. This paper proposes alternatives to implement such projects, by considering at the same time project leading, tool designing and change management.

\section{ÍNDICE}

Palavras-chave: novas tecnologias, serviços, organização do trabalho, intervenção ergonómica, prevenção

Palabras claves: nuevas tecnologías, servicios, organización del trabajo, intervención en ergonomía, prevención

Mots-clés: nouvelles technologies, services, organisation du travail, intervention ergonomique, prévention

Keywords: digitalization, services, work organization, ergonomics practice, prevention

\section{AUTORES}

\section{SYLVAIN LEDUC}

Université Aix Marseille, Laboratoire Psychologie Sociale, Aix-en-Provence, France sylvain.leduc@univ-amu.fr

\section{LUDOVIC PONGE}

SECAFI, 29 rue de l'école normale, 33200 BORDEAUX, France

ludovic.ponge@secafi.com 